\title{
COMMENTARY FROM DR NEALE FONG FCHSM
}

\author{
President Australasian College of Health Service Management
}

\section{INTRODUCTION}

As this unprecedented year of 2020 draws to a close, many of us across the health sectors globally are considering the implications of the pandemic for the way in which we work in the future. While it seems that Australia, New Zealand and some of our near neighbours have wrestled the Covid19 beast into near submission, for much of the world the battle is still unfolding. That global inequity will keep our local health systems on high alert for the coming year.

As leaders we should pause to appreciate what we have been able to achieve for our communities this year in dealing with a multitude of complex challenges.

\section{HOW WE WILL WORK}

Depending on the organisation, large swathes of the workforce are unlikely to shift back to a pre pandemic "business as usual". Most pundits suggest it will settle into a hybrid model of working where we balance the efficiencies of remote work with the social needs, creativity and innovation generated by working with others.

A key element of this shift to remote working is that managers and leaders know how to manage, coach, collaborate, evaluate performance and motivate a remote team. Another is facilitating a workplace culture of trust and belonging where interpersonal connections are fostered and prioritised. People in management roles will have to undergo a major transformation with a focus on emotional intelligence and social skills. In the past, we promoted and hired people to leadership roles based on their technical skills; in the future, we will need managers who balance their technical and people skills.

One of the key challenges for the health sector will be to transform the delivery of care at pace with the broader world of work shifts that are occurring around us. Policy and delivery will need to stay in touch with the expectations of our patients - shifts to telehealth made possible in a crisis have become a consumer expectation going forward. It is both a formidable challenge and an opportunity to enhance the efficiency of great patient care that awaits all our health leaders.

\section{TOGETHER WE CAN SUPPORT THAT FUTURE}

The College (ACHSM) has pivoted to meet the demands of virtual operations in 2020, changing many aspects of how and when we support our members. Increased and shorter briefings from health leaders via webcast presentations and panels has been welcomed by our members and critical to staying in touch. Branches have been innovative with other forms of virtual learning experiences and the Board has developed and approved a new vision of learning and development value from 2021 onwards.

With credentialling through Certification to commence on December 1, the College aims to enhance the visibility and understanding of the critical role of health leaders in the system. It is through a commitment to lifelong learning that health managers and leaders are best equipped to rise to the challenges and vicissitudes we may need to come to expect from a world where pandemics and the health impacts of climate change are a reality for the coming decades. These challenges compound with the continued need to drive efficiencies to cope with the ageing population.

Together we can support our future by developing, supporting and guiding current and future health leaders. Looking forward to 2021, the College will introduce a new online service where all members can undertake a free selfassessment against the ACHSM Master Health Service Management Competency Framework that generates an individual report identifying strengths and weaknesses. The College will publish and use the de-identified collective data to drive learning opportunities. We will also be reviewing the Competency Framework for its currency and reviewing several of our core free member offerings such 
as Mentoring. And we look forward to meeting for the National Congress in October 2021 in Canberra. Whilst we transform the way we work alongside you; we appreciate your collegiality and support as we move into a brave new future.

Dr Neale Fong FCHSM

President Australasian College of Health Service Management 